\title{
Study of Effects of Rare Earth Impurities on Structure of Matrix Emission of the Lead Tungstate Crystals
}

\author{
O. Chukova* and S. Nedilko
}

\begin{abstract}
The paper reports study of complex structure of matrix emission of undoped and RE-doped $\mathrm{PbWO}_{4}$ crystals. Decomposition of the spectra shows contributions of five components at 410, 485, 520,565, $635 \mathrm{~nm}$ in emission of all the investigated samples. Their relative contributions depend on RE doping. Observed effects of RE doping are the same for the crystals with different RE impurities. Structures of luminescent centres are discussed taking into account studied effects of the impurity $R E$ ions on spectral characteristics of the matrix emission.
\end{abstract}

Key words: lead tungstate, RE doping, luminescence.

\section{INTRODUCTION}

Luminescent properties of complex oxide compounds, and PWO is among them, are very complicated as they are related with complex structure of their crystal lattices. It is also common knowledge that defects of real crystal lattice play important role in determination of luminescent properties of complex oxides.

Emission spectra of PWO (PWO) crystals consist of several bands lying in the blue, green and orange-red spectral regions. The maximums of the noted bands are near $400-430 \mathrm{~nm}$ for the blue band, $470-520 \mathrm{~nm}$ for the green band and $550-600 \mathrm{~nm}$ for the orange-red band. Proportions of intensities of these bands depend on prehistory of the samples and experiment conditions [1, 2]. Luminescence of the PWO crystals is really caused by electron transitions in centres of different types and regular defects of crystal lattice play essential role in their formations. Therefore, application of external factors influencing on defect structure of the crystal lattice could cause remarkable changes in the luminescence properties of the PWO crystals. In this paper we consider effects of RE impurities on emission spectra of the PWO crystals.

\section{EXPERIMENTAL}

The lead tungstate crystals were grown by the Czochralski method from lead, tungsten and corresponding RE oxides $(\mathrm{RE}=\mathrm{Yb}, \mathrm{Eu}, \mathrm{Pr})$ at the Physics Faculty, Lviv National Ivan Franko University, Lviv, Ukraine, at the laboratory supervised by Prof. M. Pashkovsky.

Spectral measurements were carried out using synchrotron radiation at SUPERLUMI station at HASYLAB (DESY), Hamburg, Germany. Reflection and excitation spectra were

Physics Faculty, National Taras Shevchenko University of Kyiv, Ukraine

*hukova@univ.kiev.ua corrected using etalon sample of sodium salicylate. Emission spectra were corrected for spectral sensitivity of the registration equipment. All the spectra considered in this paper are measured at $10 \mathrm{~K}$.

\section{RESULTS AND DISCUSSION}

\section{A. Luminescent properties}

Emission spectra of the undoped PWO crystal at $10 \mathrm{~K}$ consist of the band in $350-800$ spectral range with maxima at about $460-470 \mathrm{~nm}$ at $\lambda_{e x} \leq 300 \mathrm{~nm}$. Extended long wavelength part of the spectra at these excitations evidences contribution of at least one spectral component of lower intensity with maximum near $570 \mathrm{~nm}$. Spectra obtained at $\lambda_{e x}$ $\geq 309 \mathrm{~nm}$ consist of the bands in $420-800 \mathrm{~nm}$ spectral range those have maxima at about 520 and $570 \mathrm{~nm}$, besides the long wavelength side of the spectra contains component with maximum near $600 \mathrm{~nm}$ (Fig. 1). Intensities of the bands with maxima at $460-470 \mathrm{~nm}$ are higher by one order of magnitude than intensities of the noted above long wavelength bands.

Incorporation of the $\mathrm{Yb}, \mathrm{Eu}$ or Pr impurities in the PWO lattice shifts position of the main band to the short wavelength region $\left(445 \mathrm{~nm}\right.$ at $\lambda_{e x}=270 \mathrm{~nm}$ ). Effects of various RE ions are similar. Spectra of the Yb-doped are presented on Fig. 1. As the spectra of matrix emission of the undoped and doped crystals are expected to consist of the same wide band components, comparison of their spectra allows estimate locations of these components. Comparing spectra recorded at various excitations, we consider that low temperature spectra of matrix emission could be formed by five components with maximum positions at about 400,470 , $520,570,630 \mathrm{~nm}$.

\section{B. Decomposition of the spectra}

Decomposition of spectra into elementary components requires conversion of the measured spectra from wavelength to energy scale. The conversion from the fixed wavelength bandwidth $(\mathrm{dW})$ to energy bandwidth $(\mathrm{dE})$ needs intensity conversion from $\mathrm{I}(\mathrm{W}) \mathrm{dW}$ to $\mathrm{I}(\mathrm{E}) \mathrm{dE}$. Therefore, for equivalent conversion the intensities of the converted spectra were corrected by $\lambda^{2}$ factor. Procedures of decomposition of the corrected spectra were carried out using Gauss functions and standard mathematical programs. Maximum positions of the obtained components of decomposition somewhat differ from the one noted above obtained after analytical analysis of the spectral shapes. All the obtained spectra are formed by bands with maximum positions at 410, 460, 505, 565 and $635 \mathrm{~nm}$. Results of decomposition are collected in Table 1 for all the investigated samples. 


\section{Effects of RE doping}

The obtained under decomposition spectral bands are correspondent with main types of emission considered previously for the PWO crystals. Below we shortly consider effects of RE ions on each of the bands of decomposition using results accumulated in Table 1.

The blue band at $410 \mathrm{~nm}(3.0 \mathrm{eV})$. The RE impurities lead to essential increasing of relative contribution of this band. This band is caused by exciton emission [2,3]. We consider that impurity RE ions form additional RE-induced channel of exciton creation [4].

The blue-green band at $460 \mathrm{~nm}(2.7 \mathrm{eV})$. Relative contribution of this bans insignificantly falls down in the RE-doped samples. This band arises from the $\mathrm{WO}_{4}{ }^{2-}$-type excitons in the lead deficient sheelite-type structure [5]. Adding of the RE ions decreases content of lead vacancies in the PWO lattice by filling of the $\mathrm{Pb}$ vacancies. Therefore, our results confirms conclusions concerning arising of this emission from the $\mathrm{WO}_{4}{ }^{2-}$-type excitons in the lead deficient sheelite-type structure.

The green band at $505 \mathrm{~nm}(2.45 \mathrm{eV})$. We have observed different effects of the applied impurity RE ions on spectral properties of this band (Tables 1-4). Relative contribution of the green band doesn't depend on presence of impurities at excitation from the $309-325 \mathrm{~nm}$ spectral range, but these centres considerably lose excitation energy within its transfer to the impurity RE centres at $\lambda_{e x} \leq 300 \mathrm{~nm}$. Such result agrees with obtained previously data on thermal stability of the $\mathrm{WO}_{3}$ centres [5].

The yellow band at $565 \mathrm{~nm}(2.2 \mathrm{eV})$. In general, relative contribution of this band does not depend on presence of the RE impurities. Such observation agrees with formerly made assumption that this emission is caused by transitions in interstitial oxygen anions [2].

The red band at $635 \mathrm{~nm}(1.95 \mathrm{eV})$. Adding of the RE ions strongly decreases relative intensity of this band. It was already shown previously that centres of the red emission are connected with the lead vacancies [6].

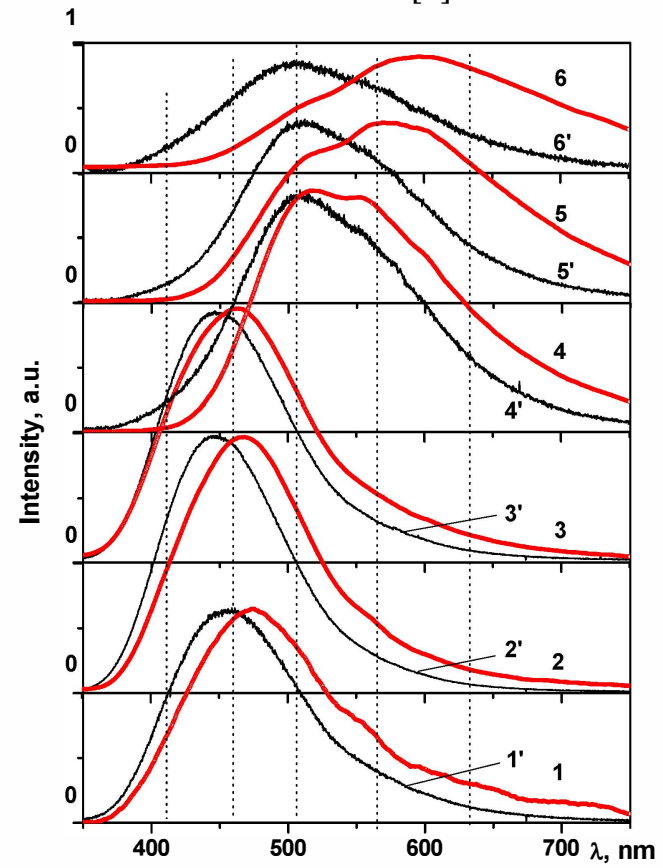

Fig. 1. Emission spectra of the undoped $(1-6)$ and Yb-doped $\left(1^{\prime}-6\right.$ ' PWO crystals, $\lambda_{e x}=175$ (1), 270 (2), 300 (3), 309 (4), 321 (5), 334 (6) nm.
Table 1. Relative intensities of components of emission spectra of the PWO crystals (normalized on the total maximum for each spectrum)

\begin{tabular}{|c|c|c|c|c|c|c|}
\hline \multirow[t]{2}{*}{ Crystal } & \multirow{2}{*}{$\lambda_{e x}, \mathrm{~nm}$} & \multicolumn{5}{|c|}{ Band positions, eV (nm) } \\
\hline & & $\begin{array}{l}1.95 \\
(635)\end{array}$ & $\begin{array}{l}2.2 \\
(565)\end{array}$ & $\begin{array}{l}2.45 \\
(505)\end{array}$ & $\begin{array}{l}2.7 \\
(460)\end{array}$ & $\begin{array}{l}3.0 \\
(410)\end{array}$ \\
\hline \multirow[t]{6}{*}{ undoped } & 334 & 0.65 & 0.5 & 0.5 & 0.1 & - \\
\hline & 321 & 0.4 & 0.6 & 0.8 & 0.1 & - \\
\hline & 309 & 0.2 & 0.5 & 0.9 & 0.1 & - \\
\hline & 300 & 0.05 & 0.1 & 0.3 & 0.65 & 0.6 \\
\hline & 270 & - & 0.1 & 0.3 & 0.75 & 0.4 \\
\hline & 175 & 0.1 & 0.1 & 0.4 & 0.7 & 0.5 \\
\hline $\mathrm{Yb}^{3+}-$ & 334 & 0.15 & 0.35 & 0.7 & 0.5 & 0.3 \\
\hline \multirow[t]{5}{*}{ doped } & 321 & 0.1 & 0.4 & 0.8 & 0.3 & 0.1 \\
\hline & 309 & 0.05 & 0.4 & 0.8 & 0.35 & 0.15 \\
\hline & 300 & - & 0.05 & 0.2 & 0.7 & 0.8 \\
\hline & 270 & - & 0.05 & 0.15 & 0.6 & 0.8 \\
\hline & 175 & - & 0.05 & 0.2 & 0.8 & 0.7 \\
\hline $\mathrm{Eu}^{3+}-$ & 321 & 0.1 & 0.55 & 0.8 & 0.15 & - \\
\hline \multirow[t]{5}{*}{ doped } & 317 & 0.1 & 0.55 & 0.8 & 0.15 & - \\
\hline & 297 & - & 0.1 & 0.25 & 0.65 & 0.5 \\
\hline & 270 & - & 0.1 & 0.25 & 0.7 & 0.5 \\
\hline & 175 & 0.05 & 0.15 & 0.4 & 0.7 & 0.5 \\
\hline & 82 & 0.1 & 0.4 & 0.6 & 0.5 & 0.7 \\
\hline $\mathrm{Pr}^{3+}-$ & 321 & 0.2 & 0.25 & 0.75 & 0.3 & - \\
\hline \multirow[t]{5}{*}{ doped } & 297 & - & 0.05 & 0.2 & 0.6 & 0.7 \\
\hline & 284 & - & 0.05 & 0.2 & 0.6 & 0.7 \\
\hline & 270 & - & 0.05 & 0.2 & 0.6 & 0.7 \\
\hline & 220 & 0.05 & 0.1 & 0.3 & 0.6 & 0.6 \\
\hline & 175 & 0.05 & 0.1 & 0.4 & 0.6 & 0.7 \\
\hline
\end{tabular}

\section{Conclusions}

Measured spectra of matrix emission of the undoped and RE-doped PWO crystals consist of the same five bands. Incorporation of the RE ions increase intensity of the $410 \mathrm{~nm}$ band by additional RE-induced channel of exciton creation and strongly decreases relative intensity of the $635 \mathrm{~nm}$ emission by reducing content of the lead vacancies. Relative contribution of the $565 \mathrm{~nm}$ band does not depend on the RE impurities presence. Intensities of the 460 and $505 \mathrm{~nm}$ bands depend on RE doping insignificantly.

\section{ACKNOWLEDGMENT}

Luminescent measurements were performed using excitation with synchrotron radiation at SUPERLUMI station (Beamline I) at HASYLAB (DESY) in Hamburg, Project Nr. I-20110592.

\section{REFERENCES}

[1] S. Zazubovich and M. Nikl, "Photo- and thermally stimulated luminescence of non-stoichiometric undoped $\mathrm{PbWO}_{4}$ crystals", Phys. Stat. Sol. (b), vol. 247, pp. 385-392, 2009.

[2] S.G. Nedilko, A.S. Voloshinovskii, M.O. Krisjuk, Z.T. Moroz and M.V. Pashkovskyi, "Impure and defect lead tungstate single crystals: X-ray and photoluminescence properties", Proceedings, pp. 263-266, 1996 [International Conference on Inorganic Scintillators and Their Applications, August 28-September 1, 1995].

[3] M. Itoh and T. Sakurai, "Time-resolved luminescence from JahnTeller split states of self-trapped excitons in PbWO4", Phys. Rev. B, vol. 73 , pp. 235106,2006

[4] O. Chukova and S. Nedilko, "Study of RE-impurity effects on exciton luminescence of $\mathrm{PWO}_{4}$ single crystals grown by Czochralski method", Optical Materials, vol. 35, pp. 1735-1740, 2013.

[5] V Babin, P. Bohachek, A. Krasnikov, M. Nikl, A. Stolovits and S. Zazubovich, "Origin of green luminescence in $\mathrm{PbWO}_{4}$ crystals", $J$. of Luminescence, vol. 124, pp. 113-115, 2007

[6] P. Bohacek, N. Senguttuvan, V. Kiisk, A. Krasnikov, M. Nikl, I. Sildos, Y. Usuki and S. Zazubovich, "Red emission of $\mathrm{PbWO}_{4}$ crystals", Radiation Measurements, vol. 38, pp. 623-626, 2004. 